\title{
De novo Splice Site Mutation of the CHD7 Gene in a Chinese Patient with Typical CHARGE Syndrome
}

\author{
Shujuan Wang Ying Lin Pengfei Liang Qiong Li Wei Li Zhaoxia Wang \\ Jian Wang Jun Chen Dingjun Zha \\ Department of Otolaryngology and Head and Neck Surgery, Xijing Hospital, Air Force Military Medical University, \\ Xi'an, PR China
}

\author{
Keywords \\ CHARGE syndrome - Congenital abnormalities - CHD7 . \\ Minigene assay $\cdot c .3523-2 A>G$ variant
}

\begin{abstract}
Introduction: CHARGE syndrome (CS, OMIM 214800) is a rare genetic disease characterized by multiple congenital abnormalities, including coloboma, heart defect, atresia of the choanae, retardation of development, genital anomalies, and ear anomalies/deafness. The syndrome is mainly caused by a heterozygous variant in the chromodomain helicase DNA-binding protein 7 (CHD7) gene that encodes the CHD7 protein, involved in the ATP-dependent remodeling of chromatin. Methods: In this study, the next-generation sequencing targeted panel was used to detect a de novo variant c.3523-2A $>\mathrm{G}$ in the $\mathrm{CHD7}$ gene in a patient with severe $\mathrm{CS}$, congenital heart disease, left coloboma of the choroid, cryptorchidism, and congenital deafness. The Sanger sequencing confirmed the variant and clarified it as de novo variant by short tandem repeat analysis in the patient family. We analyzed the effect of a variant by Minigene assay to evaluate the pathogenicity of the variant. Results: In summary, CDNA analysis confirmed that c.3523-2A > G variant activates a cryptic splice site, resulting in 172 base pair missing in exon 15 , leading to the premature truncation of the CHD7
\end{abstract}

karger@karger.com www.karger.com/orl

Karger $\stackrel{\text { ' }}{5}$

GOPEN ACCESS
(C) 2022 The Author(s)

Published by S. Karger AG, Basel

This is an Open Access article licensed under the Creative Commons Attribution-NonCommercial-4.0 International License (CC BY-NC) (http://www.karger.com/Services/OpenAccessLicense), applicable to the online version of the article only. Usage and distribution for commercial purposes requires written permission. protein (p.V1175Afs*11). Conclusion: The present study functionally characterized the novel c.3523-2A>G variant in $C H D 7$, providing further confirmatory evidence that it is associated with CS.

(c) 2022 The Author(s)

Published by S. Karger AG, Basel

\section{Introduction}

CHARGE syndrome (CS, OMIM 214800) is a rare disease with multiple anomalies that have been estimated to occur from 1:8,500 to 1:12,000 live births worldwide [1]. It was first reported and described by Hall [2], while in 1981 Pagon et al. [3] used the English acronym CHARGE to refer to the disease, which stood for the main clinical manifestations of the disease: coloboma, heart defects, atresia of the choanae, retardation of growth and development, genital hypoplasia, ear and hearing abnormalities. At present, the CS diagnosis mainly depends on clinical symptoms, while the molecular diagnosis is an important addition to the diagnosis. CS is an autosomal dominant congenital disorder that is mainly caused by $\mathrm{CHD} 7$ gene heterozygous pathogenic variants.

Shujuan Wang and Ying Lin contributed equally to this work.
Correspondence to:

Dingjun Zha, zhadjun@fmmu.edu.cn 
CHD7 gene, which is located at chromosome 8 (8q12), has a genomic size of $188 \mathrm{~kb}$ and consists of 38 exons, encodes chromodomain helicase DNA-binding (CHD) protein 7. The CHD7 has an 8,994-base pair (bp) open reading frame and 938 pathogenic variants (American College of Medical Genetics and Genomics). There are several types of mutations, including nonsense mutations, frameshift causing deletions or insertions, splice sites, missense mutations, larger deletions and duplication, translocations, and small in-frame deletions [4]. Heterozygous mutations and deletions of $C H D 7$ are found in $60-70 \%$ of patients [5, $6]$. The mild patients with only a few symptoms can transmit their mutations and have offspring [7]. Family histories of CS are rare as most variants are unique for a patient or family [4], and $97 \%$ of $C H D 7$ variants are de novo [8].

In this article, next-generation sequencing (NGS)-targeted panel was used to detect a de novo variant c.3523$2 \mathrm{~A}>\mathrm{G}$ in the $\mathrm{CHD} 7$ gene in a patient with severe CS. With a short tandem repeat (STR) analysis of the patient family, we clarified it as the de novo variant. To evaluate the pathogenicity of the variant, we analyzed the effect of the variant by Minigene assay. Our results suggested that the alteration induces the premature truncation of the CHD7 protein (p.V1175Afs*11), thus resulting in CS.

\section{Materials and Methods}

\section{Patient and Clinical Assessment}

Parents were interviewed to collect the following information: family history, the health of the mother during pregnancy, the age of onset, and clinical history of the patient, as well as the operations the patient underwent.

\section{DNA Extraction}

A total of $3 \mathrm{~mL}$ of peripheral venous blood was collected from the proband and his parents and brother. With EDTA anticoagulation, FlexGen Blood DNA Kit (CWBIO, Beijing, China) was used to extract and purify genomic DNA from whole blood. NanoQ Spectrophotometer (CapitalBio, Beijing, China) was used to determine the DNAs purity and concentration. Appropriate samples were diluted to a $100 \sim 200 \mathrm{ng} / \mathrm{L}$ concentration, and the rest was stored at $-20^{\circ} \mathrm{C}$.

\section{Next-Generation Sequencing}

An NGS-targeted panel covering exonic regions and nearby flanking regions of 168 sensorineural hearing loss (SHL)-associated genes were used. Using an S220 Focused-ultrasonicator (Covaris, Woburn, MA, USA), the gDNA of $3 \mu \mathrm{g}$ was cut to an average size of 150-bp fragment. With DNA Sample Prep Reagent Set (MyGenostics, Beijing, China), the standard Illumina libraries were prepared. The targeted genes were captured using the GenCap deafness capture kit (MyGenostics Inc.). The enrichment libraries were sequenced on NextSeq 500 (Illumina, San Diego, CA, USA) sequencer for paired-reading of $150 \mathrm{bp}$. The work was completed by Beijing Mckino Medical Institute.

\section{Bioinformatics Analysis}

Data analysis and bioinformatics processing were conducted step-by-step. First, data preprocessing was carried out to remove the adapter, low-quality reads, and short sequences. BWA software (http://bio-bwa.sourceforge.net/) compared the pretreated data with the human genome (H. sapiens, hg19, GRCh37, 02.2009). Samtools (http://samtools.sourceforge.net/) and Picard (http:// www.psc.edu/index.php/user-resources/software/picard) were used to make these data applicable for GATK (https://www. broadinstitute.org/gatk/) software [9]. GATK software detected single-nucleotide variants, single-nucleotide polymorphism, and insertion-deletion. The ANNOVAR (http://annovar.openbioinformatics.org/en/latest/) tool annotated the data. PolyPhen-2 [10] (http://genetics.bwh.harvard.edu/pph2/) and SIFT [11] (http:// sift.jcvi.org) were used to predict whether single-nucleotide polymorphism-altered proteins would affect functions. To establish the potential impact on the splicing mechanism, missense variants were analyzed using the Human Splicing Finder (HSF) software [12] (http://www.umd.be/HSF3/). The pathogenicity of each mutation site was strictly analyzed according to American College of Medical Genetics and Genomics guidelines.

\section{Molecular Diagnosis}

We designed specific primers for the $C H D 7$ gene exon 15 and adjacent intronic regions (forward primer: $5^{\prime}$-CATTGTCTTGTCCСТCTTCTCTA-3'; reverse primer: $5^{\prime}$-GGAGACAGGAATGACTATACACC- $\left.3^{\prime}\right)$. PCR amplifications were performed with DNA from the proband and his family. $100 \mathrm{ng}$ DNA was used for each PCR reaction, with $1 \times$ the Golden Star T6 Super Mix (Tsingke Biological Technology, Beijing, China) and $10 \mu \mathrm{M}$ of each primer. After an initial denaturation for $2 \mathrm{~min}$ at $98^{\circ} \mathrm{C}, 35$ cycles of amplification were carried out as follows: $98^{\circ} \mathrm{C}$ for $10 \mathrm{~s}, 58^{\circ} \mathrm{C}$ for $15 \mathrm{~s}$, and $72^{\circ} \mathrm{C}$ for $15 \mathrm{~s}$. The final extension was performed at $72^{\circ} \mathrm{C}$ for $5 \mathrm{~min}$.

After the amplified fragments were purified and Sanger sequenced on a Genetic analyzer (3130xl; ABI, Waltham, MA, USA), the work was completed by Tsingke Biological Technology. Sequencing profiles were compared with the CHD7 reference sequence Gene ID: 55636 (https://www.ncbi.nlm.nih.gov/ gene/55636) using the MegAlign multiple sequence alignment tool.

\section{Short Tandem Repeat Analysis}

The amplification of STRs was used to confirm the correct parent-child relationship. We screened 16 reported genes STR markers spanning D8S1179, D21S11, D7S820, CSF1PO, D3S1358, D5S818, D13S317, D16S539, D2S1338, D19S433, vWA, D12S391, D18S51, D6S1043, and D6S1043FGA with AmpF/STRTM HuaXiaTM Kit (Applied Biosystems by Thermo Fisher Scientific) on ABI Genetic Analyzer 3500 (Applied Biosystems). Genotypes were assigned with GeneMapper software (Applied Biosystems).

\section{Minigene Assay}

To analyze the de novo CHD7 splice variant (c.3523-2A>G), in vitro splicing assay was carried out. Briefly, wild-type (WT) and mutant (MT) CHD7 included section intron 13, exon 14, intron 14 , exon 15 , and section intron 15 , which were PCR amplified from a control individual and the proband with specific primers (forward primer: $5^{\prime}$-TGGATCTGTCTACCTGACAC- $3^{\prime}$ and reverse primer: $5^{\prime}$-GTATGGCTGGCACTACACTG-3'). The PCR reac- 
Fig. 1. a Pedigree map. Molecular diagnosis of the patient by classical Sanger sequence screening of the $\mathrm{CHD} 7$ gene: detection of the de novo c.3523-2A>G mutation. $\mathbf{b}$ WT in parents and brother. $\mathrm{c} M \mathrm{MT}$ in the patient. WT, wild type; MT, mutant type.

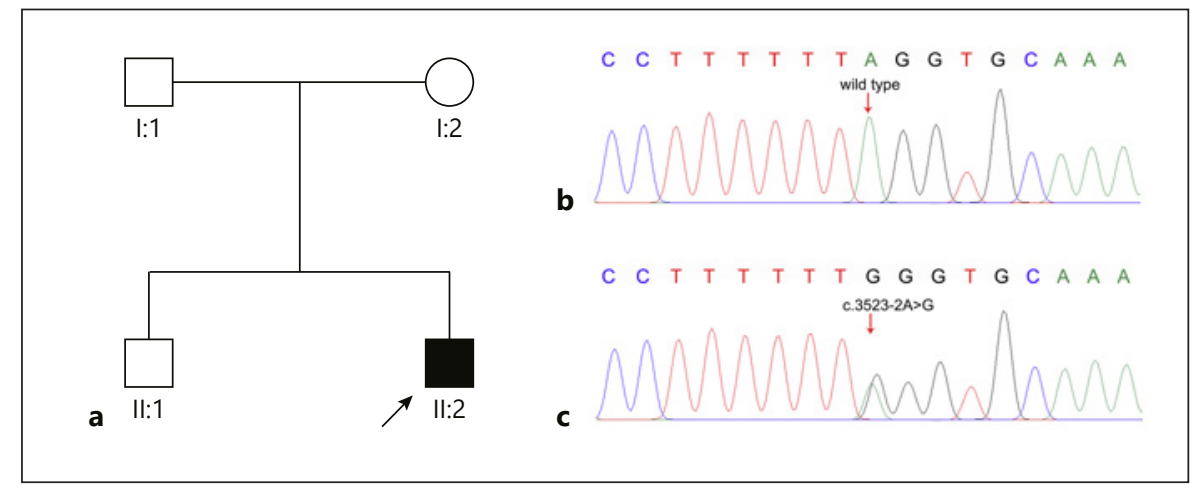

tion amplified fragment was 5,225 bp. We added NotI and, NdeI restriction site to the primer separately (forward primer with Not $\mathrm{I}$ restriction site: $5^{\prime}$-ACCAGAATTCTGGAGCTCGAGCGGCCGC- $3^{\prime}$ and reverse primer with $N d e$ I restriction site: $5^{\prime}-\mathrm{CCA}$ AACATTATGTACCTCTGTATCATATG-3'). After PCR amplification, PCR clean-up, restriction enzyme digestion of the PCR fragments, and pSPL3 exon trapping vector were performed before cloning fragments into the linearized pSPL3-vector and DH5a competent cells; WT and MT-containing vector sequences were confirmed by Sanger sequencing. Vectors containing either homozygous or WT sequence were then transfected into African green monkey kidney COS7 cells (BEINGEN, Hangzhou, China). An empty vector and negative transfection reactions were included as controls. The transfected cells were harvested $48 \mathrm{~h}$ after transfection for RNA extraction. Total RNA was prepared using miRNA easy Mini Kit (Qiagen, Venlo, The Netherlands). Approximately 1 $\mu \mathrm{g}$ of RNA was reverse transcribed using a High Capacity RNA-tocDNA Kit (Applied Biosystems) following the manufacturer's protocols. The cDNA was PCR amplified using vector-specific SD6 forward $\left(5^{\prime}\right.$-TCTGAGTCACCTGGACAACC- $\left.3^{\prime}\right)$ and reverse SA2 $\left(5^{\prime}\right.$-ATCTCAGTGGTATTTGTGAGC- $\left.3^{\prime}\right)$ primers. The amplified fragments were visualized on a $1 \%$ agarose gel, and subsequently, Sanger sequenced.

\section{Results}

\section{Clinical Presentation}

Herein, we described the case of a male toddler born at 39 weeks and 2 days by cesarean delivery. Aural deformity and left small palpebral fissure were detected soon after birth. Clinical examination showed umbilical hernia, occult penis, and incomplete orchiocatabasis. He underwent chest-enhanced CT and vascular reconstruction $\mathrm{CT}$ at 4 months due to recurrent choking and abundant expectoration. Imaging indicated congenital heart disease and narrowed bronchus, as the arch of the aorta descended to the right combined with Kommerel diverticulum and patent ductus arteriosus. The distal part of the right upper lobe bronchus, distal part of the left main

De novo Splice Site Mutation of the CHD7 Gene bronchus, the proximal part of the right middle lobe, and proximal part of the left lower lobe were all narrowed.

He was hospitalized because of fever and pneumonia at 8 months. The 2-dimensional and color-Doppler assessment confirmed heart disease. Subsequently, the patient underwent vascular ring correction and ductal closure surgery, which relieved the choking problem. The fundus examination indicated the left coloboma of the choroid.

Newborn hearing screening $72 \mathrm{~h}$ after birth was abnormal. The patient was referred to our hospital at 16 months as a cochlear implantation candidate. The auditory brainstem response test showed bilateral profound hearing impairment (threshold $>97 \mathrm{dBnHL}$ ). Temporal CT detected bilateral cochlear (incomplete partition type II), bilateral semi-circular canal dysplasia. Oblique sagittal position MRI of the internal auditory canal did not identify auditory nerve bilaterally. Eight of 12 electrodes were implanted due to cochlear malformation. The intraoperative neural remote test showed no response at all. However, the patient could respond to certain sounds during the Ling sound detection test when the electrodes were open. His speech perception performance progressively improved, as confirmed by categories of auditory performance, speech intelligibility rating, infant-toddler meaningful auditory integration scale ([IT]-MAIS), and meaningful use of speech scale (MUSS) scores of 3,1,23, and 3, respectively, at 6 months post-surgery. And the categories of auditory performance, speech intelligibility rating (IT)-MAIS, and MUSS scores were 5, 3, 39 , and 32, respectively, at 24 months post-surgery. Although his growth velocity and weight gain were satisfactory, he could not independently walk at 24 months. No clinical characteristics of CS were detected in the patient's parents and brother.

\section{Identification of a de novo CHD7 Variant}

Exome sequencing and bioinformatics analysis of 168 SHL-associated genes were performed. After variant anno- 

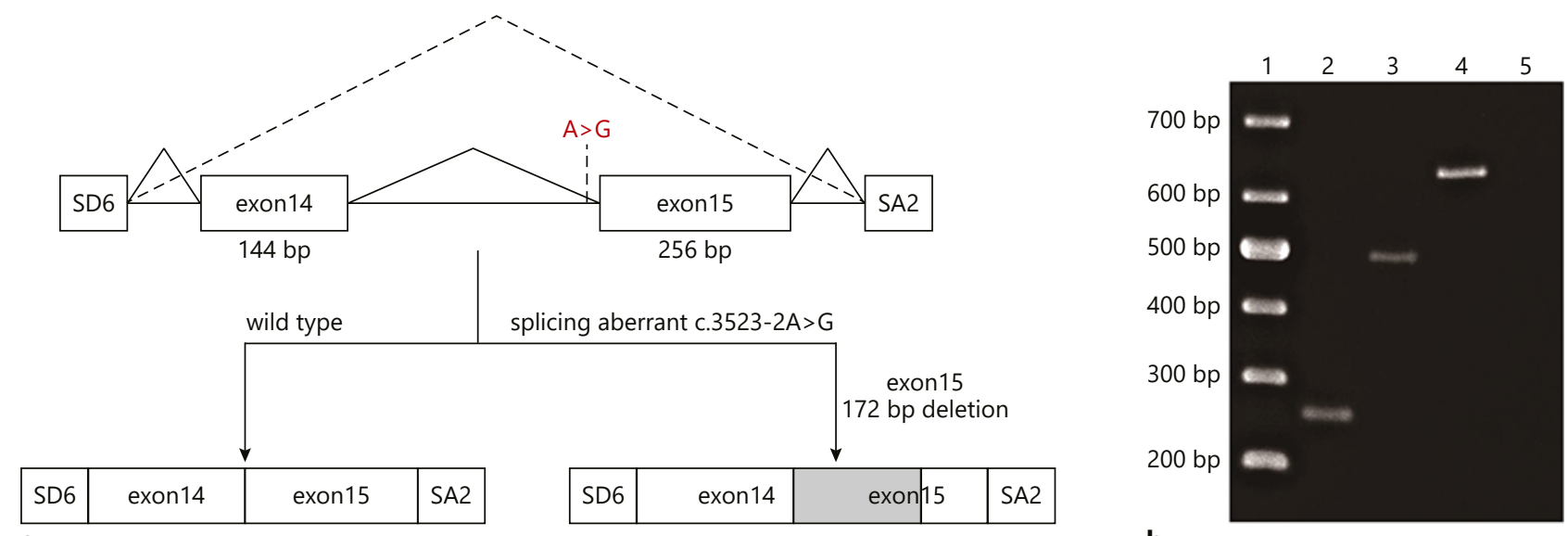

a

wild type

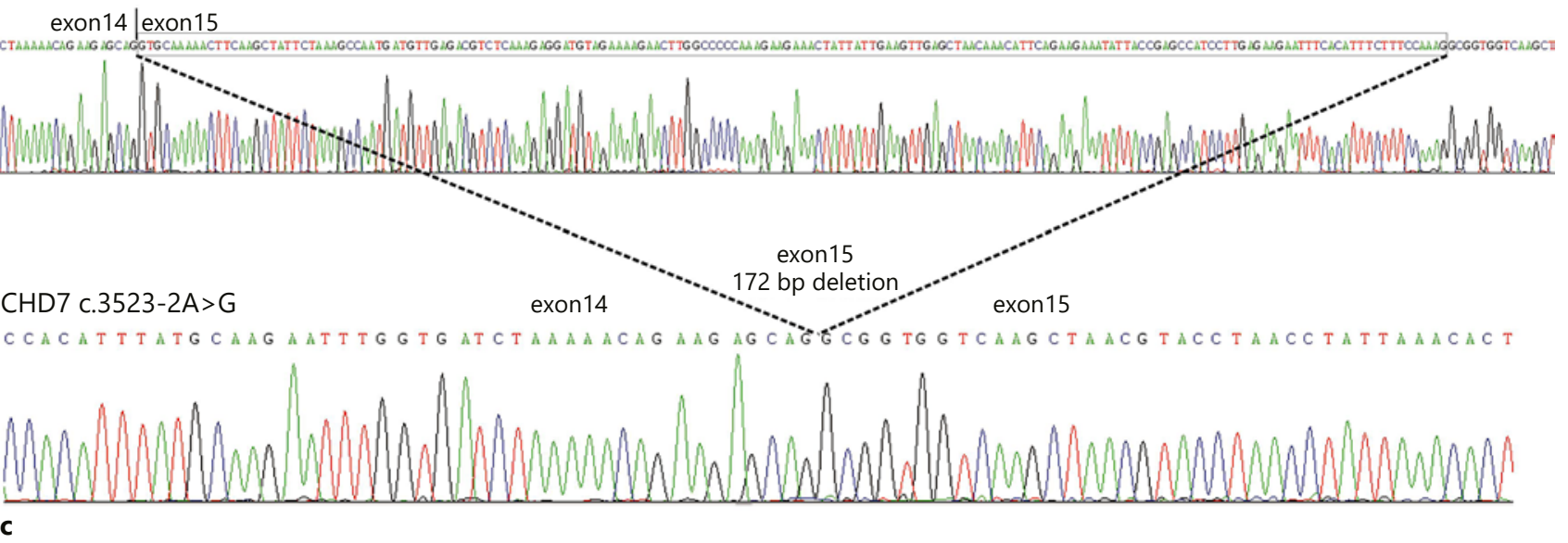

Fig. 2. Structure and functional analysis of the minigene CHD7. a A schematic of the pSPL3 exon trapping vector with cloned CHD7 part of the intron 13-exon 14-intron 14-exon 15-part of the intron 15. Plasmids were inserted into the vector in the DH5a cell. b Plasmids were transfected into African green monkey kidney COS7 cells. The plasmids transcription into RNA in the African green monkey kidney COS7 cells. The cDNA was PCR amplified using vector-specific SD6 forward (5'-TCTGAGTCACCTGGACAACC- $3^{\prime}$ ) and reverse SA2 (5'-ATCTCAGTGGTATTTGT-

tation and filtering steps on the SHL customized panel, de novo variants were detected in the CHARGE-related gene. The proband revealed a heterozygosis $C H D 7$ (NCBI Reference Sequence: NM_017780) splice variant c.3523-2A>G that abolished the acceptor splice site. This mutation was not present in dbSNP138, 1000 Genomes Project, ESP6500, and Human Gene Mutation Database professional. The
GAGC-3') primers. The amplified fragments were visualized on a $1 \%$ agarose gel. 1 : Marker; $2: \mathrm{NT}=263 \mathrm{bp} ; 3: \mathrm{NT}+\mathrm{MT}=491 \mathrm{bp}$; 4: NT + WT $=663$ bp. c The Sanger sequencing of WT and MT splicing reporter minigenes using vector-specific primers. The WT is 400 bp with complete exon 14 and exon 15. The MT c.3523$2 \mathrm{~A}>\mathrm{G}$ is 228 bp with complete exon 14 and part of exon 15 with 172 bp missing. NT, nontransfected; WT, wild type; MT, mutant type; $\mathrm{CHD7}$, chromodomain helicase DNA-binding protein 7; PCR, polymerase chain reaction.

mutation was also not present in the Human Gene Mutation Database professional (http://www.hgmd.org/) or ClinVar (http://www.ncbi.nlm.nih.gov/clinvar/), suggesting it was a de novo mutation. No other potentially pathogenic single-nucleotide variants and CNVs were detected in genes possibly responsible for CS [4]. Human Splicing Finder software analysis showed that it was pathogenic. 
Fig. 3. a Sequence alignment produced by ClustalO of the CHD7 protein in 9 species. The CHD7 sequences of the 9 species were obtained from the NCBI database. b A schematic representation of the wild type and mutated $\mathrm{CHD} 7$ protein. The mutation produces a truncated protein with 10 altered amino acids (position 1175-1184: AVVKLTYLTY) and a premature STOP codon at position 1185, missing double BRK domain, SANT domain, helicase C domain, and part of SNF2 domain.

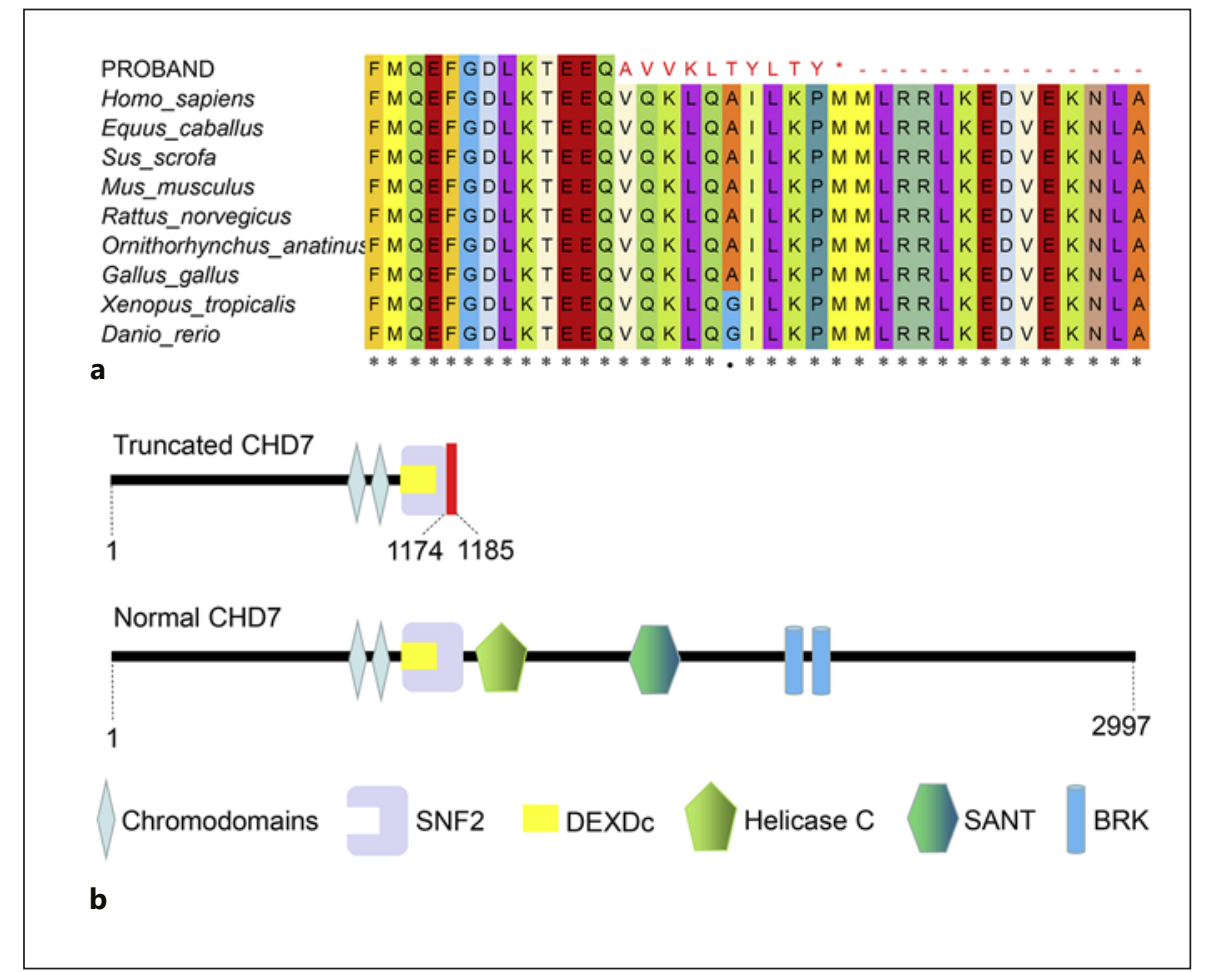

DNA screening of the proband and his family for the splice variant c.3523-2A $>\mathrm{G}$ was performed with the Sanger sequencing. The results confirmed a heterozygous mutation in the proband and WT in his parents and brother (Fig. 1).

\section{STR Analysis}

The correct parent-child relationship is very important in the identification of spontaneous gene mutation in the proband. The genotype analysis of STRs confirmed the paternity of $99.99 \%$. These findings indicated that the mutation c.3523-2A $>\mathrm{G}$ was a new spontaneous mutation.

Functional Analyses of the Splice Variant c.3523-2A $>G$

The effect of the de novo CHD7 gene c.3523-2A>G splicing variant was studied with a minigene assay that revealed 172 bp deletion in MT (Fig. 2a). PCR amplification and agarose gel electrophoresis of the empty vector's endogenous, WT, and MT showed 3 different fragments (Fig. 2b). Sanger sequencing of WT and MT cDNA generated 2 different sequences (Fig. 2c). WT control had $400 \mathrm{bp}$ fragments with complete exon 14 and exon 15. Due to the activation of a cryptic site in exon 15, $172 \mathrm{nu}-$ cleotides were deleted; thus, MT had a 228-bp fragment. The $172 \mathrm{bp}$ deletion leads to a frameshift, and created a premature termination codon (TAA) nucleotides down- stream (p.V1175Afs*11). In silico, with missing double BRK domain, SANT domain, helicase $\mathrm{C}$ domain, and part of SNF2 domainthe, the truncated $\mathrm{CHD} 7$ protein was only $40 \%$ of the full protein (Fig. $3 \mathrm{~b}$ ). The multiple CHD7 amino acid sequences alignment in 9 species revealed that the region containing the splice site variant is highly conserved among vertebrate species (Fig. 3a).

\section{Discussion and Conclusion}

SHL is caused by a variety of damage sources that impair hair cell and spiral ganglion neuron function, including mutations in deafness genes [13, 14], aging, noise exposure, chronic cochlear infections and ototoxic drugs $[15,16]$. Including sensorineural hearing loss, CS is a rare autosomal dominant multisystem disorder that usually results from alterations in one allele of the $\mathrm{CHD} 7$ gene. As CS has a close resemblance to several other genetic disorders, genetic testing should be an important tool for patients with clinical suspicion [17]. CHD7 (OMIM: 608892) encodes CHD7 on chromosome 8q12, which is a kind of ATP-dependent chromatin remodeling enzyme. With the domain and its helicase function, $\mathrm{CHD} 7$ could adjust and control the dyeing quality and remodeling. $\mathrm{CHD} 7$ is essential for the formation of multipotent migratory neu- 
ral crest cells [18]. Moreover, it can also indirectly impact the interaction between the neural crest and other tissues [4]. During early embryonic development, $\mathrm{CHD} 7$ is highly expressed in many tissues and organs such as a neural tube, undifferentiated neuroepithelium, and stroma, from which neural crest originates. Common abnormal tissues and organs in CS are neural tubes and neural crest cell derivatives, which could explain the pleiotropic nature of the phenotypic spectrum of CHARGE mutations [19]. Both haploinsufficiency, caused by loss-of-function alleles, and dominant-negative forms of the protein are believed to be responsible for the CHARGE phenotype $[18,20]$.

This study detected a de novo c.3523-2A $>$ G variant in the CHD7 gene in a patient with severe CS. The c.3523-2A $>\mathrm{G}$ variant led to abnormal gene expression. Assuming that a protein is translated, the truncated protein only contains the chromodomain, helicase $\mathrm{N}$, DEXDc, and part of SNF2 in the N-terminal [21-23]. Only $40 \%$ of the full protein is generated. Due to insufficient protein doses, the patient suffers from an umbilical hernia, occult penis, and incomplete orchiocatabasis, congenital heart disease and narrowed bronchus hearing impairment, cochlear malformation, and left choroidal defect.

Using an NGS-targeted panel, a de novo splice site c.3523-2A $>\mathrm{G}$ mutation in $C H D 7$ was detected in a patient with CS. The patient's family members in this study were healthy, and the c.3523-2A $>$ G mutation was not detected with Sanger sequencing. The amplification of STRs confirmed the correct parent-child relationship. Since neither the mother nor the father resulted as carriers of the mutation, we concluded that mutation occurred de novo on one allele. The c.3523-2A $>$ G mutation detected in patient was a spontaneous splice site variant. In the CS family, only patients with mild condition may have children because of the influence of CS on gonadal hormone secretion. Also, most of the patients with CS have spontaneous mutations [8]. Commonly, cases with severe CS have no family history [7]. Splice site mutation is one of the relatively large varieties of mutations in the CHD7 gene. There are currently 123 splice site mutations recorded in the CHD7 gene, which account for $13.1 \%$ (123/938) of pathogenic mutations. Any errors during the splicing process may lead to improper intron removal, causing alterations of the open reading frame [24]. It is very challenging to predict the impact of a pathogenic splice variant [25], as these splice site alterations could skip one or more exons, or result in activation of a cryptic intraexonic donor or acceptor splice site, or the devel- opment of an intronic splice site, which may lead to aberrant inclusions of intronic parts as cryptic exons [26,27]. All the pathogenic splice variations in the $\mathrm{CHD} 7$ gene come from patients. Most of the mutation analyses from patients are primarily or exclusively performed at the genomic DNA level, while the effect of a mutation is only predicted from the primary sequence on the encoded mRNA and protein. The exact effect of a few mutations was experimentally determined by analyzing the mRNA expression and splicing patterns [28]. Only 10 splice mutations were confirmed to induce aberrant splicing using RNA-seq analysis or minigene assay, such as the c.2443$2 \mathrm{~A}>\mathrm{G}$ mutation that caused a complete skip of exon 7 with the pSPL3-CHD7 minigene [29], and the c.5405$2 \mathrm{~A}>\mathrm{G}$ variation that resulted in a shorter $\mathrm{CHD}$ 7 exon 26 with deletion 11 nucleotides [30]. Both splice sites mutations disrupting native 3 'ss induced different results to RNA precursors.

In this study, we presented the characterization of a novel heterozygous acceptor splice site c.3523-2A $>\mathrm{G}$ variant in the $C H D 7$ gene, which has not been recorded in any database. It is challenging to predict pathogenicity without mRNA or CDNA analysis for various splicing effects [31]. If the alternative splicing creates a frameshift and/or an early stop codon [26, 32-34], there is a high chance of developing a nonfunctional protein. The splice site alterations and the consequences of a DNA change, have to be evaluated at least at the RNA level by RNA/ cDNA sequencing or at the protein level using different expression systems $[26,32,34]$. As RNA was not available from patients, we used a convenient research method to study the effect of the mutation on RNA precursors by minigene assay. In the minigene assay, this variant disrupting native 3 'ss activated of cryptic acceptor splice site, produced an aberrant transcript with an in-frame deletion of 172 bp on exon 15 in RNA, and created a premature termination codon (TAA) nucleotides downstream (p.V1175Afs*11). Therefore, we hypothesized that this mutation represents a null allele, thus causing disease due to haploinsufficiency. This is consistent with previous findings suggesting that a full genetic dosage is required for the complete function of CHD7 [35].

In summary, an NGS-targeted panel was used to iden-

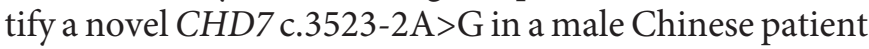
with typical CS. The minigene assay showed the mutation produced an aberrant transcript with an in-frame deletion of $172 \mathrm{bp}$, leading to a truncated protein. Further studies are needed to investigate whether the translated truncated protein can perform some functions or be directly degraded still. According to the improved survival 
in patient in this study, the earlier diagnosis of CS, the treatment will be better. Our findings provide useful information for genetic counseling and enable doctors to select appropriate clinical interventions for patients with a clear etiology of CS.

\section{Acknowledgments}

The authors wish to thank the patient's family members for their support and understanding of this work.

\section{Statement of Ethics}

The study was in compliance with the Declaration of Helsinki and approved by the Medical Ethics Commission of the First Affiliated Hospital of the Air Force Medical University (approval number KY20212002-C-1). Written informed consents were obtained from all participants or guardians before collecting peripheral blood samples of the family members.

\section{Conflict of Interest Statement}

The authors declare that they have no competing interests.

\section{Funding Sources}

This work was supported by the National Natural Science Foundation of China (81870732; 81800918), Shaanxi Provincial Science and Technology Key Project (2018PT-01), and Xijing Boost-Advanced Discipline Construction Project (XJZT14X07; XJZT18MDT07; XJZT18X23; XJZT18D16).

\section{Author Contributions}

Shujuan Wang and Ying Lin contributed to conceptualization; Shujuan Wang and Ying Lin contributed to manuscript drafting; Qiong Li and Zhaoxia Wang ascertained families and obtained clinical data; Dingjun Zha supervised the study; Pengfei Liang contributed to exome sequencing and segregation analysis; Jian Wang and Jun Chen contributed to PCR and Sanger sequencing; Wei Li provided bioinformatics support; Shujuan Wang performed Minigene analysis. All authors participated in the final review and editing of the manuscript. All authors have read and agreed to the published version of the manuscript.

\section{Data Availability Statement}

The datasets used and/or analyzed during the present study are available from the corresponding author on reasonable request. The data that support the findings of this study are openly available in NCBISequence Read Archive, and the BioProject ID is PRJNA740129 (https://www.ncbi.nlm.nih.gov/Traces/study/?acc=PRJNA740129).

\section{References}

1 Issekutz KA, Graham JM, Prasad C, Smith IM, Blake KD. An epidemiological analysis of CHARGE syndrome: preliminary results from a Canadian study. Am J Med Genet A. 2005;133A:309-17.

2 Hall BD. Choanal atresia and associated multiple anomalies. J Pediatr. 1979;95:395-8.

3 Pagon RA, Graham JM, Zonana J, Yong SL. Coloboma, congenital heart disease, and choanal atresia with multiple anomalies: CHARGE association. J Pediatr. 1981;99: 223-7.

4 Janssen N, Bergman JE, Swertz MA, Tranebjaerg L, Lodahl M, Schoots J, et al. Mutation update on the $\mathrm{CHD} 7$ gene involved in CHARGE syndrome. Hum Mutat. 2012;33: 1149-60.

5 Martin DM, Sheldon S, Gorski JL. CHARGE association with choanal atresia and inner ear hypoplasia in a child with a de novo chromosome translocation $\mathrm{t}(2 ; 7)(\mathrm{p} 14 ; \mathrm{q} 21.11)$. Am J Med Genet. 2001;99:115-9.

6 Zentner GE, Layman WS, Martin DM, Scacheri PC. Molecular and phenotypic aspects of CHD7 mutation in CHARGE syndrome. Am J Med Genet A. 2010;152A:674-86.

7 Wells C, Loundon N, Garabedian N, Wiener-Vacher S, Cordier-Bouvier MD,
Goudeffroye G, et al. A case of mild CHARGE syndrome associated with a splice site mutation in CHD7. Eur J Med Genet. 2016;59:195-7.

8 Hale CL, Niederriter AN, Green GE, Martin DM. Atypical phenotypes associated with pathogenic CHD7 variants and a proposal for broadening CHARGE syndrome clinical diagnostic criteria. Am J Med Genet A. 2016; 170A:344-54.

9 McKenna A, Hanna M, Banks E, Sivachenko A, Cibulskis K, Kernytsky A, et al. The genome analysis toolkit: a mapreduce framework for analyzing next-generation DNA sequencing data. Genome Res. 2010;20:1297303.

10 Adzhubei IA, Schmidt S, Peshkin L, Ramensky VE, Gerasimova A, Bork P, et al. A method and server for predicting damaging missense mutations. Nat Methods. 2010;7:248-9.

$11 \mathrm{Ng}$ PC, Henikoff S. Predicting deleterious amino acid substitutions. Genome Res. 2001; 11:863-74.

12 Shapiro MB, Senapathy P. RNA splice junctions of different classes of eukaryotes: sequence statistics and functional implications in gene expression. Nucleic Acids Res. 1987; 15:7155-74.
13 Guo R, Xiao M, Zhao W, Zhou S, Hu Y, Liao M, et al. 2D Ti3 C2 Tx MXene couples electrical stimulation to promote proliferation and neural differentiation of neural stem cells. Acta Biomater. 2020. Epub ahead of print.

14 Qi J, Liu Y, Chu C, Chen X, Zhu W, Shu Y, et al. A cytoskeleton structure revealed by superresolution fluorescence imaging in inner ear hair cells. Cell Discov. 2019;5:12.

15 He ZH, Zou SY, Li M, Liao FL, Wu X, Sun HY, et al. The nuclear transcription factor FoxG1 affects the sensitivity of mimetic aging hair cells to inflammation by regulating autophagy pathways. Redox Biol. 2020;28:101364.

16 Liu Y, Qi J, Chen X, Tang M, Chu C, Zhu W, et al. Critical role of spectrin in hearing development and deafness. Sci Adv. 2019;5: eaav7803.

17 Siavriene E, Petraityte G, Mikstiene V, Rancelis T, Maldziene Z, Morkuniene A, et al. A novel CHD7 variant disrupting acceptor splice site in a patient with mild features of CHARGE syndrome: a case report. BMC Med Genet. 2019;20:127.

18 Bajpai R, Chen DA, Rada-Iglesias A, Zhang J, Xiong Y, Helms J, et al. CHD7 cooperates with PBAF to control multipotent neural crest formation. Nature. 2010;463:958-62. 
19 Sanlaville D, Verloes A. CHARGE syndrome: an update. Eur J Hum Genet. 2007;15:389-99.

20 Bergman JE, Janssen N, Hoefsloot LH, Jongmans MC, Hofstra RM, van Ravenswaaij-Arts CM. CHD7 mutations and CHARGE syndrome: the clinical implications of an expanding phenotype. J Med Genet. 2011;48:334-42.

21 Hall JA, Georgel PT. CHD proteins: a diverse family with strong ties. Biochem Cell Biol. 2007;85:463-76.

22 Marfella CG, Imbalzano AN. The CHD family of chromatin remodelers. Mutat Res. 2007; 618:30-40.

23 Kim HG, Kurth I, Lan F, Meliciani I, Wenzel $\mathrm{W}$, Eom SH, et al. Mutations in CHD7, encoding a chromatin-remodeling protein, cause idiopathic hypogonadotropic hypogonadism and Kallmann syndrome. Am J Hum Genet. 2008;83:511-9.

24 Anna A, Monika G. Splicing mutations in human genetic disorders: examples, detection, and confirmation. J Appl Genet. 2018;59: 253-68.

25 Katoh-Fukui Y, Yatsuga S, Shima H, Hattori A, Nakamura A, Okamura K, et al. An unclassified variant of $\mathrm{CHD} 7$ activates a cryptic splice site in a patient with CHARGE syndrome. Hum Genome Var. 2018;5:18006.
26 Chen LL, Sabripour M, Wu EF, Prieto VG, Fuller GN, Frazier ML. A mutation-created novel intra-exonic pre-mRNA splice site causes constitutive activation of KIT in human gastrointestinal stromal tumors. Oncogene. 2005;24:4271-80.

27 Krawczak M, Reiss J, Cooper DN. The mutational spectrum of single base-pair substitutions in mRNA splice junctions of human genes: causes and consequences. Hum Genet. 1992;90:41-54.

28 Lopez-Bigas N, Audit B, Ouzounis C, Parra G, Guigo R. Are splicing mutations the most frequent cause of hereditary disease? FEBS Lett. 2005;579:1900-3.

29 Lee B, Duz MB, Sagong B, Koparir A, Lee KY, Choi JY, et al. Revealing the function of a novel splice-site mutation of CHD7 in CHARGE syndrome. Gene. 2016;576:776-81.

30 Legendre M, Rodriguez-Ballesteros M, Rossi $\mathrm{M}$, Abadie V, Amiel J, Revencu N, et al. CHARGE syndrome: a recurrent hotspot of mutations in CHD7 IVS25 analyzed by bioinformatic tools and minigene assays. Eur J Hum Genet. 2018;26:287-92.
31 Wang GS, Cooper TA. Splicing in disease: disruption of the splicing code and the decoding machinery. Nat Rev Genet. 2007;8:74961.

32 Caminsky N, Mucaki EJ, Rogan PK. Interpretation of mRNA splicing mutations in genetic disease: review of the literature and guidelines for information-theoretical analysis. F1000Res. 2014;3:282.

33 Ferreira PG, Oti M, Barann M, Wieland T, Ezquina S, Friedländer MR, et al. Sequence variation between 462 human individuals fine-tunes functional sites of RNA processing. Sci Rep. 2016;6:32406.

34 Ipe J, Swart M, Burgess KS, Skaar TC. HighThroughput assays to assess the functional impact of genetic variants: a road towards $\mathrm{Ge}$ nomic-Driven medicine. Clin Transl Sci. 2017;10:67-77.

35 Bergman JE, Janssen N, van der Sloot AM, de Walle HE, Schoots J, Rendtorff ND, et al. A novel classification system to predict the pathogenic effects of $\mathrm{CHD} 7$ missense variants in CHARGE syndrome. Hum Mutat. 2012;33: 1251-60. 